\title{
Neonatal intramural calcification associated with jejunal atresia: a rare case report
}

\author{
Chakraborty G. ${ }^{1}$, Das S. ${ }^{2}$, Sugandhi N. ${ }^{3}$, Jadhav A. ${ }^{4}$, Acharya S.K ${ }^{5}$, Bagga D. ${ }^{6}$ \\ ${ }^{1}$ Dr. Goutam Chakraborty, Assistant Professor, ${ }^{2}$ Dr. Sumanta Das, PG Trainee, Department of Pathology, ${ }^{3}$ Dr. Nidhi Sugandhi, \\ Associate Professor, ${ }^{4}$ Dr. Amit Jadhav, Assistant Professor, ${ }^{5}$ Dr. S.K. Acharya, Senior Pediatric Surgeon \& HOD, ${ }^{6}$ Dr. D. Bagga, \\ Senior Consultant \& Professor; 1,3,4,5,6authors are affiliated with Department of Pediatric Surgery, Vardhman Mahavir Medical \\ College \& Safdarjung Hospital, New Delhi, India
}

Corresponding Author: Dr. Goutam Chakraborty, Assistant Professor, Department of Pediatric Surgery, Vardhman Mahavir Medical College, New Delhi, India. E-mail: drgoutam83@yahoo.com

\begin{abstract}
Neonatal intraabdominal calcifications are usually seen in conditions like intestinal atresia, meconium ileus and imperforate anus. It is mostly due to perforation of the obstructed proximal bowel followed by an inflammatory response of the matted loops and omentum, which initially is sterile to start with. Intramural calcification in intestinal atresia is a rare type of intra-abdominal calcification. The exact etiology of intramural calcification remains unclear, though ischemia secondary to any antenatal vascular insult like intussusception or volvulus is believed to be the triggering event. Intraperitoneal and intraluminal bowel calcification is generally visible on plain abdominal radiographs. Contrastingly, intramural calcification is by and large a histopathological finding. A 2-day old newborn male baby presented with signs of intestinal obstruction and was diagnosed to have jejunal atresia with meconium pseudocyst. The newborn underwent exploratory laparotomy with resection of the meconium pseudocyst and atretic and dilated part of the small bowel with jejunoileal anastomosis. Histology of dilated and atretic part of jejunum and adjacent area revealed intramural calcification.
\end{abstract}

Keywords: Jejunum, Atresia, Pseudocyst, Calcification

\section{Introduction}

Neonatal intra-abdominal calcification is a relatively rare phenomenon. Intra-abdominal calcification may occur as intraperitoneal (meconium peritonitis), intraluminal and intramural calcification $[1,2]$. Calcifications can also be seen in neonatal liver, gall bladder or adrenals. Intramural intestinal calcification is least common type among the three types of calcification. It is associated with intestinal atresia, meconium ileus, or anorectal malformations and are even seen on antenatal ultrasounds as echogenic bowel [3]. Jejunoileal atresia is an antenatally acquired reasonably rare cause of intestinal obstruction in a newborn with an

\section{Case Report}

A 2-day old, full term, male baby presented with bilious vomiting and not passing stool since birth. No abnormality was detected in antenatal ultrasounds. The baby cried immediately after birth and weighed $2.2 \mathrm{kgs}$. The baby was delivered by caesarean section.

The indication of caesarean section was fetal distress. Xray abdomen revealed abnormally dilated small bowel loops. Ultrasound findings revealed significant distension of gut loops, which suggested high bowel obstruction.

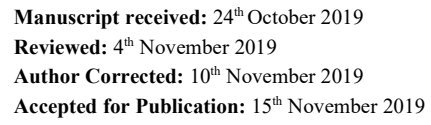

Manuscript received: $24^{\text {th }}$ October 2019

Reviewed: $4^{\text {th }}$ November 2019

Author Corrected: $10^{\text {th }}$ November 2019

Accepted for Publication: $15^{\text {th }}$ November 2019

incidence rate of 1:5000 [4,5]. It effects both sexes equally. It develops as a delayed sequelae of bowel infarction secondary to vascular insults as mentioned before. Pathologically, this is dystrophic calcification which usually conforms with the continuity of the bowel. Only a few cases of intramural calcification associated with jejunal atresias have been reported in literature. This case is presented with the objective of sensitising ourselves to the varied surgicopathological aspects of neonatal intraabdominal calcification. 


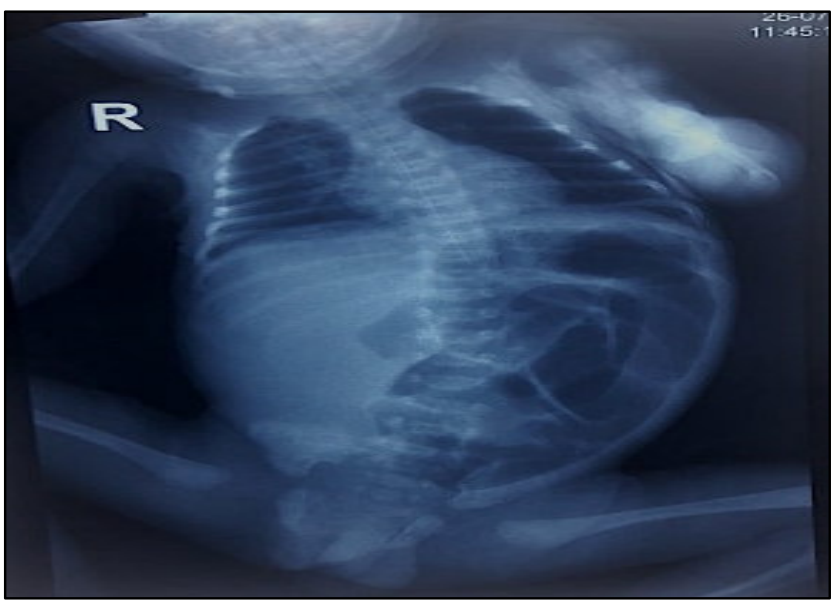

Fig-1: Dilated loops of small bowel on X-ray abdomen.

Intraoperatively the baby was diagnosed to have type-III jejunal atresia. A $4 \times 4 \mathrm{~cm}$ meconium pseudocyst was found attached to the under surface of the blind end of ileum. Multiple adhesions were found intraoperatively. $5 \mathrm{~cm}$ of jejunum, meconium pseudocyst and $3 \mathrm{~cm}$ of ileum resected and anastomosis was done. Three segments of intestine was received. One segment measured $5 \mathrm{~cm}$ in length and $2 \mathrm{~cm}$ in diameter. Resected end measures $1.3 \mathrm{~cm}$ and the other end was atretic. A perforation identified $2 \mathrm{~cm}$ from the atretic end and measured $0.8 \mathrm{~cm}$ in diameter. Section from atretic jejunum showed intramural calcification. It also revealed focal mucosal ulceration \& submucosal congestion. Section adjacent to perforation revealed mucosal ulceration, chronic inflammation \& mild serositis.

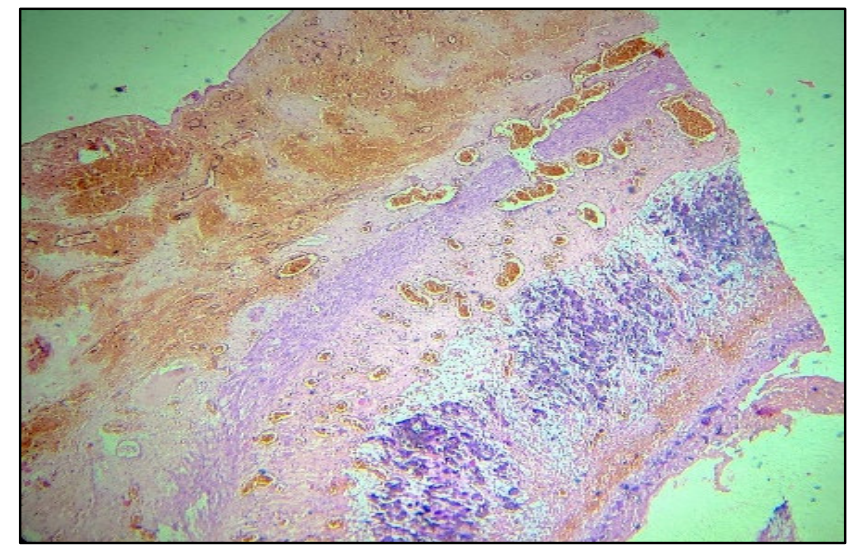

Fig-2: Intramural calcification (10X)

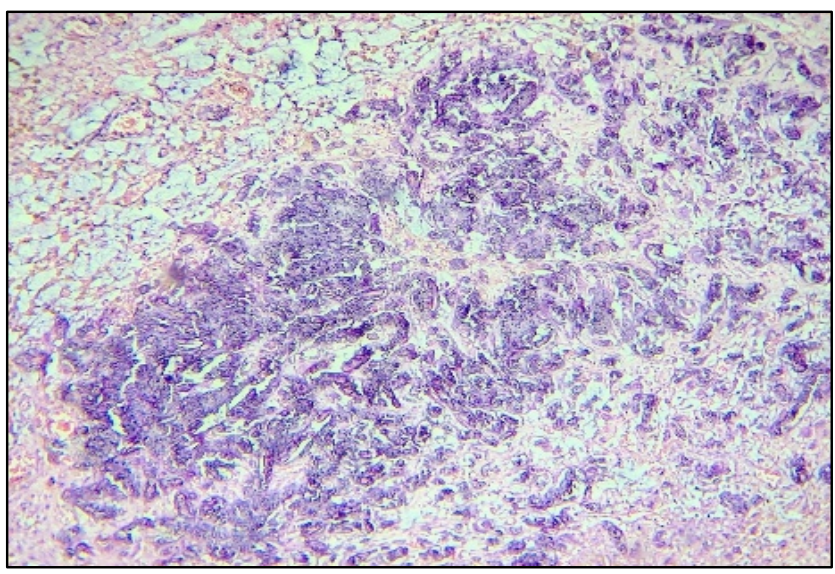

Fig-3: Intramural calcification (40X). 


\section{Discussion}

Neonatal intra-abdominal calcification is a very rare observation. However, with advanced technology ultrasound machines, these are often diagnosed in antenatal scans now a days. Historically, intraabdominal calcifications were first noted in imperforate anus patients $[1,4]$. The pathogenesis was attributed to the associated rectourinary fistula which led to complex chemical reaction between urinary metabolites like urea nitrogen and creatinine with meconium that contains mucopolysaccharides, bile salts, calcium and phosphorus [6]. This calcification was mainly intraluminal.

Radiographically, these were amorphous or punctate. The calcification of meconium peritonitis is usually linear or plaque-like on the serosal surface of the bowel and abdominal wall. Meconium peritonitis is a result of meconium spillage through perforation of the obstructed bowel wall. This causes an initially sterile peritonitis with calcium deposition composed of cornified epithelial cells, which is a constituent of extravasated meconium $[7,8]$. Papers dating back to early nineteenth century point that meconium in the peritoneal cavity can calcify within a matter of few days [9,10]. Forshall, Hall, and Rickham hypothesized that calcification in bowel wall was probably the result of intrauterine perforation of intestine [11]. Both bowel atresia and intramural calcification may be due to ischemia. Small bowel obstruction with intraluminal calcification was first described in 1949 by Camp and Roberts [5].

There, they reported a case of infant male with ileal stenosis and multiple calcified intraluminal meconium foci. There has been a report of ileal atresia associated with ileal duplication where calcification was present [7]. When analysed, a basic difference was found in pathogenesis of colonic calcification with that of small bowel calcification i.e; absence of ischemia as an inciting factor in the former. Calcifications have also been noted in neonatal solid viceras like liver (hemangiomas, mesenchymal hamartomas, etc), gall bladder (gall stones) and in adrenals (haemorrhage either spontaneous or due to birth trauma). Solid visceral calcifications may or may not have any prognostic importance in isolation. On the contrary, scattered intraperitoneal calcific deposits have been seen in antenatal bowel perforations and are universally considered as a poor prognostic factor for survival irrespective of intervention. Often these foetuses are stillborn or have a postnatal course complicated by sepsis and commands early surgery.

Literatures when reviewed mostly mention calcification which are either solid visceral, diffuse intra-abdominal or intraluminal. Intramural deposition of calcium is the rarest variant and is mostly diagnosed histologically. Intramural calcifications are reported very rarely probably because it is difficult to interprete and requires an experienced histopathologist. Limited articles do mention intramural calcification documentation in small intestine with and without atresia [1] [2]. Ours case is that of intramural calcification in jejunal atresia \& meconium pseudocyst.

Here, the patient was a 2-days-old, full term, male baby presenting with features of neonatal small bowel obstruction and later found to have jejunal atresia. This calcification may have an association with ciliary dyskinesia of cystic fibrosis [8]. However, this association was not verified due to limited resources and financial constraints.

Intramural calcification associated with small bowel atresia has been studied in a newborn within a week of birth $[2,9,10]$. Contrastingly, intramural calcification in nonatretic bowel has been documented relatively late at around 3-4weeks [6]. It points to the fact that intramural calcification may preceed intraluminal calcification. In the present case, peritoneal fluid and inter-loop adhesions of bowel loops were seen at surgery which raised the suspicion of antenatal perforation. Evidence of perforation was seen both grossly and microscopically.

Cases have been documented demonstrating calcification in the mucosa and submucosa [2,6,9]. Very frequently, an associated giant cell reaction in the submucosa has been noted [7]. This is probably due to prolonged inflammatory reaction subsequent to perforation. In the present case, the calcification was predominantly in the jejunal muscularis propria with lesser involvement of mucosa and submucosa. This is in contradiction of the fact that muscularis is the toughest layer among all, mucosa and submucosa being the most vulnerable ones. It was associated with congestion and focal erosion.

The characteristic pathological alterations in the bowel wall depend on the extent, severity, and duration of ischemic event. Jejuno-ileal atresias have always been explained as late gestational ischemic accidents in contrary to duodenal atresias. Ischemia, invariably is initiated by a vascular compromise as in intussusception, volvulus, tight abdominal wall defects (gastroschisis and omphalocele). This leads to infarctive atresia in uterus [2].

Dystrophic calcification sets in the necrotic tissue as an expected sequelae. Thus, a profound vascular accident is the inciting factor. Future course depends on the duration, site and extent of ischemia. Factors like amniotic fluid $\mathrm{Ph}$, its adequacy and placental gaseous exchange also have their influence. 


\section{Conclusion}

Intramural calcification in a case of jejunal atresia is a rare phenomenon. It can be hypothesized that vascular insult in the late gestational period is the initiating event for calcium deposition. This leads to ischemic obliteration of a segment of bowel with distal discontinuity.

The further consequences could be multifactorial and severity depends on the duration and extent of infarction. This could be the reason for variation in the site of calcific deposits-whether diffuse intraperitoneal, intraluminal or intramural. The exact etiology is still obscured and demands more elaborative studies.

Funding: No funding sources

Conflict of interest: None declared

Ethical Approval: Not Required

\section{Reference}

1. Suchitha S, Kumarguru BN, Sunila GV. Neonatal intramural calcification in jejunal atresia: Case report of a rare phenomenon. Int $\mathrm{J}$ Appl Basic Med Res. 2017; 7(4):258-260. doi: 10.4103/ijabmr.IJABMR_267_16.

2. Aharon M, Kleinhaus U, Lichtig C. Neonatal intramural intestinal calcifications associated with bowel atresia. AJR Am J Roentgenol. 1978;130(5):999-1000. doi: 10.2214/ajr. 130.5.999.

3. Vincoff NS, Callen PW, Smith-Bindman R, Goldstein RB. Effect of ultrasound transducer frequency on the appearance of the fetal bowel. J Ultrasound Med. 1999; 18(12): 799-803; quiz 805-6. doi: 10.7863 /jum. 1999. 18.12.799
4. Khilnani MT, Wolf BS, Arnheim EE. Enterolithiasis in the newborn. J Mt Sinai Hosp N Y. 1955;22(1):58-61.

5. CAMP R, ROBERTS MH. Multiple calcareous deposits in the intestinal tract of the newborn: Report of a case associated with stenosis of the ileum. Am J Dis Child. 1949; 78(3):393-400.

6. Winchester P, Heneghan M, Brill PW, Firpo A. Neonatal intramural bowel calcification without atresia. AJR Am J Roentgenol. 1981;136(4): 826-827. doi: 10.2214/ajr. 136. 4. 826

7. Sinha S, Sarin Y, Ramji S. Ileal atresia with duplication cyst of terminal ileum: a rare association. J Neonatal Surg. 2012; 1(2):27. eCollection 2012 Apr-Jun.

8. Lang I, Daneman A, Cutz E, Hagen P, Shandling B. Abdominal calcification in cystic fibrosis with meconium ileus: radiologic-pathologic correlation. Pediatr Radiol. 1997; 27(6):523-527. doi: 10.1007/s002470050172

9. Brodribb Jh. Intramural Calcification And Stenosis Of The Small Intestine Of The Newborn. Br J Radiol. 1964; 37:63-65. doi: 10.1259/0007-1285-37-433-63.

10. Vanbuskirk RW, Kurlander Gj, Samter Tg. Intramural Jejunal Calcification In A Newborn: A Case With Jejunal Atresia And Cystic Fibrosis. Am J Dis Child. 1965;110: 329-332. doi: 10.1001/Archpedi.1965.02090030343020.

11. Rickham PP. Intraluminal intestinal calcification in the newborn. Arch Dis Child. 1957;32(161):31-34. doi: 10. $1136 /$ adc.32.161.31.

\section{How to cite this article?}

Chakraborty G., Das S, Sugandhi N, Jadhav A, Acharya S.K, Bagga D. Neonatal intramural calcification associated with jejunal atresia: a rare case report. Trop J Path Micro 2019;5(11):962-965.doi:10.17511/jopm.2019.111.21 